\title{
Changes in spectral parameters of corneal pulse following canaloplasty
}

\author{
Monika E. Danielewska ${ }^{1}$ (1) - Aleksandra K. Kicińska ${ }^{2} \cdot$ Michał M. Placek $^{1} \cdot$ Katarzyna Lewczuk $^{2} \cdot$ Marek Rękas $^{2}$
}

Received: 8 May 2019 /Revised: 9 July 2019 / Accepted: 23 July 2019/Published online: 3 August 2019

(C) The Author(s) 2019

\begin{abstract}
Purpose To ascertain whether changes in the spectral content of the corneal pulse (CP) signal, measured in vivo in primary openangle glaucoma (POAG) patients, indirectly reflect changes in corneal biomechanics after canaloplasty.

Methods Fifteen eyes of 15 POAG patients who underwent canaloplasty combined with phacoemulsification were enrolled. Standard ophthalmic examinations were conducted before washout, pre-operatively, at days 1, 7, and 1,3, 6, and 12 months after surgery. Non-contact measurements of the CP signal were performed at pre-washout, pre-operatively, and at 3, 6, and 12 months post-operatively. Then, amplitudes of the $\mathrm{CP}$ first five harmonics associated with the heart rate were estimated. Temporal changes of all considered parameters were tested at a Bonferroni-adjusted significance level set to 0.005 .

Results A decrease in the amplitude of the first harmonic and an increase in the normalized amplitude of the third harmonic $\left(A_{\mathrm{CP} 3 \mathrm{n}}\right)$ of the $\mathrm{CP}$ signal were noticed between the pre-washout and the pre-operative stages $(p=0.003$ and $p=0.004$, respectively). This corresponds to an increase in median intraocular pressure (IOP) values by $6.0 \mathrm{mmHg}$ $(p=0.0045)$. After surgery, $A_{\mathrm{CP} 3 \mathrm{n}}$ reached the highest value at 3 months post-operatively, compared with pre-washout level $(p=0.0045)$.

Conclusions Alterations in corneoscleral stiffness caused by surgery are reflected in changes in the $A_{\mathrm{CP} 3 n}$ value. Hence, postoperative corneal biomechanics could be monitored indirectly by this supporting indicator that can be used to estimate the time at which measures of IOP are no longer biased by the changed cornea boundary conditions caused by canaloplasty.

Clinical Trials Registration NCT02908633
\end{abstract}

Keywords Corneal biomechanics · Glaucoma surgery · Spectral analysis · Ocular pulse

\section{Introduction}

Canaloplasty is a modern and safer alternative to traditional surgeries used for surgical treatment of open-angle glaucoma [1]. The advantage of canaloplasty over filtering surgeries is the lack of subconjunctival bleb and hence no need for antimetabolites, rare post-operative complications, and uncomplicated follow-up [2]. The purpose of canaloplasty is

Monika E. Danielewska

monika.danielewska@pwr.edu.pl

1 Department of Biomedical Engineering, Faculty of Fundamental Problems of Technology, Wrocław University of Science and Technology, Wybrzeże Wyspiańskiego 27, 50-370 Wrocław, Poland

2 Department of Ophthalmology, Military Institute of Medicine, ul. Szaserów 128, 04-141 Warsaw, Poland to restore natural trabeculocanalicular outflow pathways by catheterizing and viscodilating the entire length of Schlemm's canal as well as placing a 10-0 Prolene suture with the use of flexible microcatheter [3]. Canaloplasty is characterized by a high short- and long-term safety profile and high efficiency in the sustained intraocular pressure (IOP) reduction in adult patients with open-angle glaucoma [3-5]. Although proved to be safer than trabeculectomy, which is still regarded as the gold standard, canaloplasty has less pronounced IOP-lowering potential $[6,7]$.

Static evaluation of IOP is usually the standard parameter for monitoring long-term efficacy of canaloplasty [8-10]. However, it is well known that IOP undergoes cyclic fluctuations due to eye dynamics [11]. Also, the biomechanical properties of ocular tissues affect the IOP measurement [12]. Recently, more attention has been paid to finding parameters complementary to IOP for better assessing the outcome of glaucoma surgery, particularly the parameters describing the 
aforementioned dynamic nature of the eye. Rękas and colleagues [13] were first to report that 24-h fluctuations pattern of corneoscleral limbus area (CSLA), measured with the Sensimed Triggerfish contact lens sensor in glaucoma patients, can be clinically useful for assessing mid-term efficacy of canaloplasty. Those findings emphasize the significance of characteristics of the eye dynamics in monitoring glaucomatous eyes after surgeries aiming at IOP reduction. CSLA changes, reflecting the ocular pulse (OP), undergo not only diurnal processes related to the circadian rhythm but also those linked to the heart rhythm. The dynamics of CSLA and OP characteristics may have a diagnostic potential that is worth exploring.

OP is a highly complex phenomenon reflecting the dynamic behavior of the eye. It manifests itself in ocular volume changes according to the heart rhythm and includes signals of natural pulsatile variations in IOP [11, 14, 15], ocular blood flow $[16,17]$, aqueous humor secretion and outflow [18, 19], and movements of anterior [14, 20-22] and posterior eye tissues [23-26]. Some of OP signals have found their origins in the systemic circulation [11, 14-16, 27], and their parameters have been shown to be correlated with cardiovascular activity [28-31]. Further, OP signals have also been considered in studying pathological conditions of the eye, such as glaucoma, $[24,32,33]$, underlining the role of ocular biomechanical properties in disease processes.

In the current study, we focus particularly on the corneal pulse (CP), which is an OP manifestation representing a superposition of longitudinal eye movements and slight semi-periodic corneal surface expansion $[14,20,21]$. The $\mathrm{CP}$ is closely related to both cardiovascular activity $[30,31]$ and corneal biomechanics [34]. The CP signal can be acquired in a simple way, without disturbing the natural movements of the cornea, using a non-invasive and non-contact ultrasonic technique $[21,22,35]$. This method has recently been used to measure corneal pulsation in elderly subjects [22] and glaucoma patients [36]. As a result, the morphology of the $\mathrm{CP}$ signal has been shown to change with age [22] and in glaucoma [36], pointing at potential higher ocular stiffness in those patients. Spectral analysis of OP signals has proved to be useful for differentiating glaucoma patients from healthy subjects $[37$, 38]. Hence, further investigation of the $\mathrm{CP}$ characteristic in frequency domain could provide more insight into natural corneal expansion and ocular rigidity, and offer independent and supportive parameters to standard ophthalmological ocular parameters with clinical utility in glaucoma.

The purpose of this study was to ascertain whether changes in spectral parameters of the $\mathrm{CP}$ signal, acquired in vivo, provide indirect information on corneal biomechanics after canaloplasty.

\section{Materials and methods}

\section{Study design}

Fifteen Caucasian patients with uncontrolled mild to moderate primary open-angle glaucoma (POAG) and cataract were enrolled in this study. All patients underwent canaloplasty combined with phacoemulsification. Additionally, a reference age-matched group of 15 healthy subjects was included.

At the time of qualification, all participants underwent past and current general medical as well as ophthalmic history review, including pharmacological treatment and surgical interventions. Exclusion criteria were narrow-angle or secondary glaucoma, neovascular disease, uveitis, peripheral anterior synechiae, any corneal abnormalities, history of angle recession or trauma, previous antiglaucoma surgery or laser therapy, and high myopia (spherical equivalent greater than 6D). Patients with a previous history of IOP greater than $30 \mathrm{mmHg}$ were also excluded, based on the observation made in an animal ex vivo study that distal aqueous outflow pathway may be irreversibly collapsed in such individuals [39]. Only one eye per patient was eligible.

The study was approved by the Bioethics Committee of the Military Institute of Medicine in Warsaw (decision no. 67/WIM/2015) and adhered to the tenets of the Declaration of Helsinki. Before any measurements, the purpose of the study and the study protocol were explained to the patients, who were also informed about the surgical alternatives. After declaring participation in the study for at least 12 months, all participants signed a consent form. The trial was registered at clinicaltrials.gov before recruitment (registration no. NCT02908633).

\section{Pre-operative protocol and surgical technique}

Patients qualified for combined cataract and glaucoma surgery were scheduled for a washout period of 4 weeks before surgical intervention. Before washout, all patients underwent baseline ophthalmic examination including best-corrected visual acuity (BCVA), IOP measurement with Goldmann tonometry (GAT), gonioscopy, slit-lamp, and fundus examination. Additionally, ultrasound pachymetry (DGH-550 Pachette 2, DGH Technology, Inc., Exton, PA, USA) and optical biometry (IOL-Master, Carl Zeiss Meditec AG, Jena, Germany) were performed. At the pre-operative stage, GAT was obtained and only patients with post-washout IOP of $18 \mathrm{mmHg}$ or higher were scheduled for surgery. Monocular visual field (VF) testing was performed with the 24-2 algorithm of the Humphrey Field Analyzer (Carl Zeiss Meditec AG, Germany) prior to surgery. 


\section{Surgical technique}

Surgical procedures have been performed under retrobulbar anesthesia (2\% xylocaine and $0.5 \%$ bupivacaine) by one surgeon (M. R.). First, a fornix-based conjunctival flap was dissected to expose sclera. This was followed by creating scleral flaps, as described previously by Lewis et al. [40], and Bellucci and Morseli [41]. A $5.0 \mathrm{~mm} \times 5.0 \mathrm{~mm}$, one-third scleral thickness, parabolic in shape, the superficial flap was extended forward into the clear cornea and a 1.0$\mathrm{mm}$ smaller, in size, the deep flap was dissected to expose trabeculo-Descemet's membrane (TDM) and reach Schlemm's canal. At this stage, $2.2 \mathrm{~mm}$ clear corneal phacoemulsification was performed using the Infiniti Vision System (Alcon Surgical, Fort Worth, TX) followed by an IOL implantation. After completing the TDM, the inner wall of Schlemm's canal was removed and the deeper scleral flap was excised. Catheterization around the entire circumference of the canal was carried out with a standard canaloplasty set (iTrack from Ellex Medical Lasers Pty Ltd., Adelaide, Australia). Once the distal tip was exposed, a 10-0 polypropylene suture was tied to it and pulled into the canal. With careful withdrawing of microcatheter under control of an illuminating beacon tip, viscoelastic was injected every $2 \mathrm{~h}$. After viscodilation, the tip was exposed at the ostium and the suture was cut off and knotted under tension in order to distend the trabecular meshwork inward. After the excision of the deep flat, the superficial flap was sutured in a watertight manner over intrascleral space, which resulted in creating a scleral lake. The conjunctival flap was fixed to the corneal limbus.

\section{Post-operative protocol}

Post-operative examinations were performed at days 1 and 7 , and 1, 3, 6, and 12 months post-operatively, and whenever it seemed clinically necessary. It included BCVA testing, GAT, slit-lamp biomicroscopy for anterior segment assessment, gonioscopy with angle grading, and indirect funduscopy. Pachymetry and optic biometry measurements were taken at 3, 6, and 12 months. At all follow-up examinations, patients were reviewed for the number and type of antiglaucoma medications; adverse events were also recorded.

Two definitions of success were used: complete and qualified. Complete surgical success was defined as IOP $\leq$ $18 \mathrm{mmHg}$ with no antiglaucoma medications, and qualified success was defined as IOP $\leq 18 \mathrm{mmHg}$ with or without medications. A procedure was considered to be a failure when IOP $>18 \mathrm{mmHg}$ with or without glaucoma medication, or when further glaucoma surgery was required.

\section{Corneal pulse measurement}

Additionally, at pre-washout, on the day of surgery (pre-op), and at 3, 6, and 12 months after surgery, non-contact measurements of the CP signal were performed by using an ultrasonic transducer $[35,42]$ that was placed in a specially constructed holder mounted on a slit-lamp. To minimize the movements of the patient's head, a belt was used that strapped the head to the ophthalmic headrest frame. The single $\mathrm{CP}$ recording took $10 \mathrm{~s}$ and was repeated five times for the eye classified to canaloplasty. Synchronously with the CP signal, the blood pressure (BP) signal was measured using a CNAP Monitor 500 (CNSystems Medizintechnik GmbH, Graz, Austria).

\section{Data analysis}

Estimation of spectral parameters of the $\mathrm{CP}$ and BP signals was performed in a custom program written in MATLAB (MathWorks, Inc., Natick, MA, USA). First, raw CP and BP signals were preprocessed numerically, including linear trend removal and band-pass filtering in the range from 0.6 to $20 \mathrm{~Hz}$ to eliminate the frequencies related to respiration modulation (below $0.6 \mathrm{~Hz}$ ) and those not related to the fundamental frequencies of the heart activity. Then, for each patient and at each visit, the first three pairs (CP, BP) of signals that were not affected by eye blink artifacts or substantial head movements in the CP signal, as evaluated by an expert from the signal trend, were selected for further analysis. For each BP signal, the mean arterial pressure (MAP) and systolic (SBP) and diastolic (DBP) blood pressures were computed. Then, the representative mean values of MAP, SBP, and DBP were calculated across the three selected repetitions of BP signals for each patient and at each visit.

Signals spectra were computed using the Fourier transform. Since a 10-s-long BP signal can be assumed stationary (spectral content not changing in time) [30], its amplitude spectrum was used to estimate the frequency $f_{\mathrm{BP} 1}$ of the first harmonic (the fundamental frequency) associated with the heart rate. Then, the corresponding first harmonic, $f_{\mathrm{CP} 1}$, was determined in the CP spectrum in the range of $f_{\mathrm{BP} 1} \pm 0.2 \mathrm{~Hz}$. For $f_{\mathrm{CP} 1}$ and its four subsequent harmonics, amplitudes $A_{\mathrm{CP} 1}, A_{\mathrm{CP} 2}, A_{\mathrm{CP} 3}$, $A_{\mathrm{CP} 4}$, and $A_{\mathrm{CP} 5}$ were calculated. Next, for each CP recording, amplitudes of its second to fifth harmonics were normalized to the amplitude of the first harmonic, giving $A_{\mathrm{CP} 2 \mathrm{n}}, A_{\mathrm{CP} 3 \mathrm{n}}$, $A_{\mathrm{CP} 4 \mathrm{n}}$, and $A_{\mathrm{CP} 5 \mathrm{n}}$, where the subscript $\mathrm{n}$ denotes normalization. Finally, the representative mean values of $A_{\mathrm{CP} 1}, A_{\mathrm{CP} 2 \mathrm{n}}$, $A_{\mathrm{CP} 3 \mathrm{n}}, A_{\mathrm{CP} 4 \mathrm{n}}$, and $A_{\mathrm{CP} 5 \mathrm{n}}$ were calculated across the three selected repetitions of the $\mathrm{CP}$ signals for each patient and at each visit.

For each patient's visit, the mean values from the three repeated measurements of IOP were calculated. For prewashout as well as post-operatively, analogous means were calculated also for CCT, ACD, and AL. Visual acuity in 
Snellen equivalents was converted to $\log$ MAR units (logarithm of minimum angle of resolution).

\section{Statistical analysis}

The Kolmogorov-Smirnov test was used to assess the null hypothesis of data normality and there were no reasons to reject it for demographic data, IOP, ocular biometric, and blood pressure parameters for POAG patients at the prewashout stage and for healthy subjects $(p>\alpha=0.05, \alpha$ being the significance level). Therefore, an independent-samples $t$ test was used to compare those parameters between POAG patients and the healthy group.

Averaged spectral parameters, IOP values, ocular parameters, and blood pressure parameters for POAG patients after the pre-washout stage generally did not follow a normal distribution. Hence, temporal changes of the abovementioned parameters were tested using the Wilcoxon signed rank test with Bonferroni corrected $\alpha=0.005$. Temporal analyses did not include the healthy group, which was only used as a reference for the pre-washout stage of the POAG group.

Partial correlations, with time set as a control variable, were applied between the CP signal parameters, IOP and blood pressure parameters, separately for data before (pre-operative stage) and after surgery (post-operative stage). The calculations were performed in SPSS 22.0 (SPSS, Inc., Chicago, IL, USA).

\section{Results}

Demographic data and ocular and blood pressure parameters for POAG patients and healthy subjects are summarized in Table 1 with the corresponding results of $t$ test. Note that no statistically significant differences were found between POAG patients and the healthy reference group in all considered parameters.

In the time period between the pre-washout and the pre-op stage - across which the cornea demonstrates natural (that is, without surgical incision) biomechanical changes with the IOP increase - the median value of IOP increased by $6 \mathrm{mmHg}$ (Wilcoxon test, $p<0.001$ ) (see Tables 2, 3, and Fig. 1). At 3 months after canaloplasty - when changes in corneal biomechanics governed by different boundary conditions occur - the median value of IOP decreased statistically significantly by $9 \mathrm{mmHg}$ with respect to the pre-op stage $(p<0.001)$ and then remained at almost the same level up to 12 months post-operatively (see Fig. 1). The complete or
Table 1 Patients' demographics and the considered set of parameters for the POAG group at the pre-washout stage and a reference healthy group with the results of the independentsamples $t$ test

\begin{tabular}{|c|c|c|c|}
\hline Data & $\begin{array}{l}\text { POAG group } \\
\text { Mean } \pm \mathrm{SD} \text { (range) }\end{array}$ & Healthy group & $t$ test \\
\hline Age (years) & $\begin{array}{l}73 \pm 8 \\
(59-87)\end{array}$ & $\begin{array}{l}69 \pm 4 \\
(64-75)\end{array}$ & $t(28)=1.671, p=0.10$ \\
\hline Sex (female/male) & $9 / 6$ & $9 / 6$ & - \\
\hline Eye (right/left) & $3 / 12$ & $4 / 11$ & - \\
\hline IOP (mmHg) & $\begin{array}{l}16.9 \pm 2.7 \\
(12.0-21.0)\end{array}$ & $\begin{array}{l}15.5 \pm 1.6 \\
(13.0-18.0)\end{array}$ & $t(28)=1.769, p=0.088$ \\
\hline $\mathrm{CCT}(\mu \mathrm{m})$ & $\begin{array}{l}531 \pm 33 \\
(481-584)\end{array}$ & $\begin{array}{l}526 \pm 33 \\
(466-574)\end{array}$ & $t(28)=0.368, p=0.715$ \\
\hline $\mathrm{ACD}(\mathrm{mm})$ & $\begin{array}{l}3.13 \pm 0.39 \\
(2.53-3.90)\end{array}$ & $\begin{array}{l}2.90 \pm 0.35 \\
(2.22-3.48)\end{array}$ & $t(28)=1.746, p=0.092$ \\
\hline $\mathrm{AL}(\mathrm{mm})$ & $\begin{array}{l}23.50 \pm 1.14 \\
(21.76-25.83)\end{array}$ & $\begin{array}{l}22.88 \pm 0.84 \\
(21.54-24.32)\end{array}$ & $t(28)=1.695, p=0.101$ \\
\hline $\mathrm{MD}(\mathrm{dB})$ & $\begin{array}{l}-5.29 \pm 3.05 \\
(-0.91 \text { to }-13.29)\end{array}$ & - & - \\
\hline $\operatorname{PSD}(\mathrm{dB})$ & $\begin{array}{l}5.27 \pm 2.72 \\
(1.25-9.19)\end{array}$ & - & - \\
\hline $\mathrm{SBP}(\mathrm{mmHg})$ & $\begin{array}{l}152 \pm 22 \\
(128-183)\end{array}$ & $\begin{array}{l}141 \pm 19 \\
(102-166)\end{array}$ & $t(23)=1.426, p=0.167$ \\
\hline $\mathrm{DBP}(\mathrm{mmHg})$ & $\begin{array}{l}82 \pm 8 \\
(73-96)\end{array}$ & $\begin{array}{l}77 \pm 7 \\
(64-85)\end{array}$ & $t(23)=1.811, p=0.083$ \\
\hline MAP (mmHg) & $\begin{array}{l}108 \pm 9 \\
(91-120)\end{array}$ & $\begin{array}{l}102 \pm 12 \\
(79-116)\end{array}$ & $t(23)=1.465, p=0.156$ \\
\hline
\end{tabular}

$I O P$ intraocular pressure, $C C T$ central cornea thickness, $A C D$ anterior chamber depth, $A L$ axial length, $M D$ visual field mean deviation, $P S D$ visual field pattern standard deviation, $S B P$ systolic blood pressure, $D B P$ diastolic blood pressure, $M A P$ mean arterial pressure, $S D$ standard deviation 
qualified success rate evaluated at 12-month post-op was reached in thirteen out of fifteen patients $(\sim 86 \%)$.

Before washout, patients were taking three groups of topical medications: $\beta$-blockers ( $60 \%$ of patients), prostaglandin analogues (PGAs) (94\%), and carbonic anhydrase inhibitors $(40 \%)$, in various combinations. Six out of fifteen patients were on PGA monotherapy (latanoprost). Three patients took PGA-based fixed combination alone, whereas three patients took the same fixed combination with an adjunctive anhydraze inhibitor concomitantly. One patient used the combination of carbonic anhydrase inhibitors and $\beta$-blocker alone, whereas two patients used this combination with an unfixed combination of PGA. The median number of medications before washout was two (range one to three). At 12-month follow-up, no patient required antiglaucoma medication.

From all the ocular parameters measured, CCT, ACD, and BCVA changed significantly after 12 months post-operatively in relation to the pre-washout stage (Wilcoxon test, $p<0.001$, $p=0.003$, and $p=0.002$, respectively).

Correlation between IOP and DBP ( $\left.p=0.046, R^{2}=0.176\right)$ was found only before surgery, which can be associated with the washout of glaucoma medications.

\section{Complications}

No intra-operative complications were noted. Successful $360^{\circ}$ catheterization with the placement of a tension suture was achieved in all cases. Majority of early post-operative complications included hyphema, defined as layered blood level in anterior chamber measured after at least $1 \mathrm{~h}$ in an upright position, or microhyphema, defined as erythrocytes in the anterior chamber without layered blood (see Table 4). In all of these cases, the blood resolved within 1 week. No transient IOP elevation was noted. In one case, post-operative macular edema was diagnosed causing BCVA decrease - this resolved after topical non-steroid anti-inflammatory drugs and subtenon injection of methylprednisolone acetate.

\section{Spectral parameters of the CP signal}

At the pre-washout stage, there were no statistically significant differences in $A_{\mathrm{CP} 1}$ and $A_{\mathrm{CP} 3 n}$ between POAG patients and the healthy reference group $(t(26)=1.037, p=0.309$, and $t(26)=0.587, p=0.562$, respectively). Statistically significant differences were observed only in $A_{\mathrm{CP} 1}$ and $A_{\mathrm{CP} 3 n}$. Specifically, a decrease in $A_{\mathrm{CP} 1}$ and an increase in $A_{\mathrm{CP} 3 \mathrm{n}}$ were observed between the pre-washout and the pre-operative stage ( $p=0.003 ; p=0.004$, respectively) (see Figs. 2a and b). This corresponds to an increase in IOP values between those stages (see Fig. 1).

After surgery, when corneal biomechanics differs from that of the pre-operative stage, $A_{\mathrm{CP} 3 n}$ reaches the highest value at 3 months post-operatively in relation to the pre- 
Table 3 Results of temporal changes of IOP, ocular, and blood pressure parameters. Differences were assessed using the Wilcoxon signed rank test with the Bonferroni correction (pre-washout vs pre-op, $3 \mathrm{~m}, 6 \mathrm{~m}$, and $12 \mathrm{~m}$ post-op). Italicized values indicate $p<0.005$

\begin{tabular}{llllllllll}
\hline & Time & $\begin{array}{l}\text { IOP } \\
(\mathrm{mmHg}) \\
p \text { value }\end{array}$ & $\begin{array}{l}\mathrm{CCT} \\
(\mu \mathrm{m})\end{array}$ & ACD (mm) & AL (mm) & BCVA (logMAR) & SBP (mmHg) & DBP (mmHg) & MAP (mmHg) \\
\hline $\begin{array}{l}\text { Natural corneal } \\
\text { biomechanics }\end{array}$ & Pre-op & $<0.001$ & - & - & - & - & 0.203 & 0.799 & 0.285 \\
$\begin{array}{c}\text { Changing corneal } \\
\text { biomechanics }\end{array}$ & 3 m post-op & 0.017 & 0.220 & 0.003 & 0.010 & 0.001 & 0.441 & 0.038 & 0.173 \\
& 6 m post-op & 0.008 & 0.025 & 0.004 & 0.010 & $<0.001$ & 0.953 & 0.515 & 0.441 \\
& 12 m post-op & 0.018 & $<0.001$ & 0.003 & 0.010 & 0.002 & 0.441 & 0.314 & 0.678 \\
\hline
\end{tabular}

washout $(p=0.0045)$ and then significantly decreases at 12 months $(p=0.0045)$ (see Fig. 2b). Since nominal levels of $A_{\mathrm{CP} 1}$ and $A_{\mathrm{CP} 3 \mathrm{n}}$ values for healthy subjects are less known, those values are additionally shown in Figs. $2 a$ and $b$.

Correlation analysis with time used as a control variable showed that $A_{\mathrm{CP} 1}$ and IOP values before canaloplasty were weakly correlated $\left(R^{2}=0.174\right)$; however, this result was statistically significant $(p=0.024)$.

\section{Discussion}

Numerous earlier works widely investigated the amplitudes of OP signals, pointing at their role in the estimation of ocular rigidity $[24,32]$ and in the differentiation of glaucomatous eyes $[33,43]$. Also, spectral analysis of OP signals has shown to be a powerful tool for investigating the relationship between OP and cardiovascular activity in healthy individuals $[21,30]$, differentiating ocular hemodynamics and biomechanical properties in healthy subjects from those in glaucoma patients [25], and discriminating glaucomatous eyes from healthy eyes $[37,38,44]$. This study extends those developments to glaucoma patients undergoing canaloplasty.
To the best of our knowledge, this is the first study presenting spectral changes of $\mathrm{CP}$ signals in POAG patients treated with canaloplasty. It is revealed that the information about corneal biomechanics is indirectly carried by the spectral content of the CP signal. Hence, it has the potential to differentiate corneal biomechanics in the pre- and post-operative stages. Unlike the IOP measurement, the non-contact and noninvasive registration of the $\mathrm{CP}$ signal, on its own, is independent of the ocular biomechanical properties.

In this study, statistically significant differences in the amplitude of the first $\mathrm{CP}$ harmonic, $A_{\mathrm{CP} 1}$, and the normalized amplitude of the third CP harmonic, $A_{\mathrm{CP} 3 \mathrm{n}}$, have been observed between pre-washout and pre-op, the two stages between which the cornea demonstrates natural (that is, without surgical incision) biomechanical changes with IOP increase. Namely, the greater the IOP difference between pre-washout and pre-op stages, the smaller the $A_{\mathrm{CP} 1}$ and the higher the $A_{\mathrm{CP} 3 n}$. Our results for $A_{\mathrm{CP} 1}$ recorded in humans are in agreement with the earlier in vivo animal study of Rogala et al. [34], where the acute increase in IOP in rabbit eyes was reflected in a decrease in the power of the first $\mathrm{CP}$ harmonic. It is known that elevated IOP increases ocular rigidity [45] and that higher ocular stiffness related to the IOP elevation is expressed in
Fig. 1 Boxplots of IOP at consecutive measurement stages. $* p<0.001$

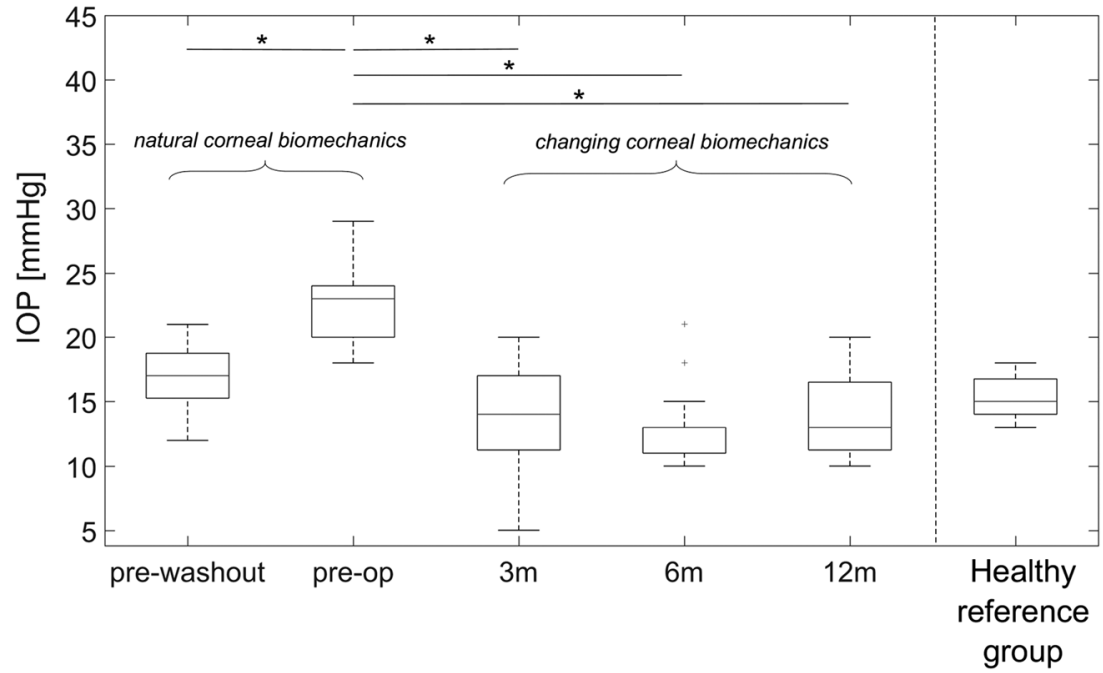


Table 4 Ocular-related postsurgical complications

\begin{tabular}{ll}
\hline Complication & Number of patients $(\%)$ \\
\hline Hyphema & $6(40.0)$ \\
Inflammatory exudates & $2(13.3)$ \\
Macular edema & $1(6.7)$ \\
IOP $\geq 30 \mathrm{mmHg}$ & none \\
Blood in vitreous & $1(6.7)$ \\
\hline
\end{tabular}

higher mechanical resistance of cornea [46]. We have demonstrated that changes in the measured IOP during the course of study alter the spectral content of the CP signal, as indicated by $A_{\mathrm{CP} 1}$ and $A_{\mathrm{CP} 3 n}$ values. However, the relationships between the IOP and the CP signal harmonics are not directly proportional, suggesting that the latter carries additional to IOP information on the ocular biomechanics.

It is important to note that our findings showed unchanged CCT values at the 3-month post-op after surgery, when IOP rapidly decreases and $A_{\mathrm{CP} 3 n}$ achieves the highest value. Therefore, we conclude that CCT has no impact on the spectral content of the CP signal. Hence, to evaluate the efficacy of canaloplasty, other biomechanical properties of cornea underlying its stiffness and biomechanically corrected IOP should be considered.

Changes in corneal biomechanics caused by the surgical incision and tensioning suture in the Schlemm's canal (SC) in canaloplasty are the contributing factors further increasing the $A_{\mathrm{CP} 3 \mathrm{n}}$ value to that achieved after the washout. The tensioning suture stiffens the limbus area changing the boundary conditions for the cornea and, subsequently, results in a damped low-frequency component $\left(A_{\mathrm{CP} 1}\right)$ and an amplified high-frequency component $\left(A_{\mathrm{CP} 3 n}\right)$ of the recorded $\mathrm{CP}$ signal. As it was shown in the earlier in vivo study with rabbits [34], biomechanical properties of the stiffer cornea (a higher Young's modulus) are reflected in a higher power of the third $\mathrm{CP}$ harmonic (around $3 \mathrm{~Hz}$ ) at normal IOP. In a way, the cornea could be viewed as a mechanical high-pass filter with gain depending on the IOP and cornea boundary conditions. Hence, the spectral parameters of the CP signal could be

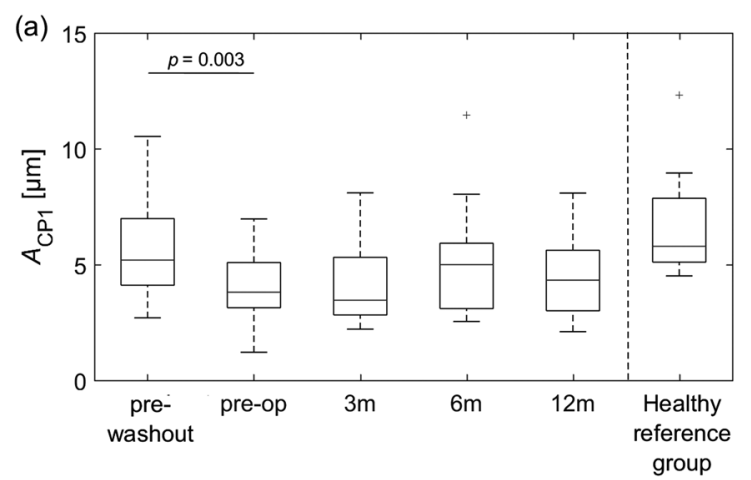

valuable factors to indirectly assess post-operative alterations in corneoscleral stiffness caused by surgeries aiming at IOP reduction.

Many studies reported that increased IOP caused a reduction in the pulsatile ocular blood flow (POBF) [46-48]. Since there is a strong correlation between the $\mathrm{CP}$ and cardiovascular activity signal parameters [14, 21, 30,31], indicating similarity of their signal spectra $[21,26,30]$, the CP signal parameters contain also information about the cardiovascular activity, apart from biomechanical properties of the cornea. In this study, POBF has not been measured directly, but the continuous blood pressure (BP) signal was registered synchronously with the $\mathrm{CP}$ signal. At the consecutive measurement stages, mean, systolic, and diastolic values of the BP signal were not correlated with the $\mathrm{CP}$ parameters, meaning no direct effect of systemic blood pressure on the CP characteristic. This outcome supports the postulate that variations in spectral parameters of the $\mathrm{CP}$ signal measured after canaloplasty are likely due to changes in corneal biomechanics.

Canaloplasty offers a very high safety profile and greater IOP reduction when combined with cataract surgery [49]. Our study confirms the earlier results of Rękas et al. [53] and Zhang et al. [54] that phacocanaloplasty decreases IOP effectively up to 12 months after surgery. Most frequent complications were hyphema or microhyphema, commonly reported in canaloplasty literature, which are considered to be a positive prognostic indicator of surgery effectiveness [50]. According to Grieshaber et al. [50], hyphema, as a result of blood reflux due to pressure gradient reversion, is to be a proof of distal aqueous outflow pathway patency. We did not record any case of persistent hypotony after surgery or choroidal or retinal detachment. No bleb-associated complications like blebitis or endophthalmitis were present. The absence of conjunctival bleb made the follow-up quicker and less demanding for patients, since no additional procedures were required.

According to the literature, dorzolamide can induce corneal edema in human eyes with borderline endothelial function [51] or corneal guttata [52], but it does not change CCT in healthy individuals. Also, the influence of using topical dorzolamide on endothelial pump function was shown in the

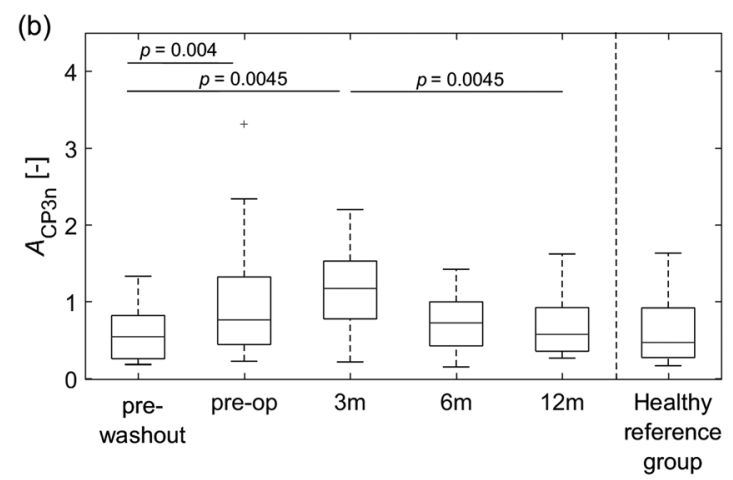

Fig. 2 Boxplots of $A_{\mathrm{CP} 1}$ (a) and $A_{\mathrm{CP} 3 n}$ (b) of the $\mathrm{CP}$ signal at consecutive measurement stages 
animal study of Teus et al. [53], where it was proved to reduce the negative intrastromal corneal pressure. Topical $\beta$-blockers have been associated with a reversible increase in CCT [54]. Prostaglandin F2 $\alpha$ (PG) analogues, on the other hand, affect corneal hysteresis, which reflects some corneal viscoelastic properties [55, 56], corneal deformation amplitude [57], and also cause a decrease in CTT with no correlation between the magnitude of CCT reduction and the reduction in IOP [58]. This effect on CCT is probably due to alteration of the corneal stromal structure by the PG F-receptor mediated synthesis of metalloproteinases [59-61] and reduction in collagen synthesis. Topical application of latanoprost also stimulates fibroblasts-mediated collagen gel contraction; thus, CCT might be affected by changes in corneal shape, as suggested by Liu et al. [62]. In clinical practice, interactions between IOP-lowering medication and the CCT are of special interest because of the risk of improper estimation of the GAT-derived IOP readings during glaucoma patients observation. In the current study, CCT increased slightly $(\sim 2 \%)$, however significantly, after 12 months post-operatively in relation to the prewashout stage, which can be an effect of surgical intervention on the corneal structure and biomechanics. On the other hand, vascular effects are the other aspect of the antiglaucoma drug action on the eye dynamics. $\beta$-blockers have been shown to reduce ocular blood flow, in particular choroidal and optic disc pulsatile blood flow [63-66]. However, in the case of other classes of drugs, contradictory results have been obtained, which could be also explained by using different measurement techniques and different treatment regimens $[63,67,68]$. No effect of antiglaucoma medications on blood flow velocities in either the central retinal or the ophthalmic artery has been observed [63, 69-73]. Furthermore, cardiovascular system can be provoked by $\beta$-blockers [74]; however, there was no measurable effect on systemic hemodynamics in healthy subjects $[63,69]$. In our study, blood pressure parameters of glaucoma patients did not differ from those of healthy subjects at the pre-washout stage, and even after washout of antiglaucoma medications, which emphasizes the lack of systemic effects. Because no systemic hemodynamic changes have been observed in our study, increased IOP should theoretically result in a decreased ocular perfusion pressure, which cannot be excluded as contributing to potential changes of choroidal blood flow. Since variations of the CP characteristic are correlated with the ocular perfusion pressure and fundus pulsation, being a valid index of pulsatile choroidal perfusion [75], druginduced changes of choroidal blood flow may be reflected in the CP characteristics as well. Based on the aforementioned studies and our own results reported here, we conclude that the increased mechanical resistance of the ocular surface after the period of washout from antiglaucoma medications, manifesting also in changes in the $\mathrm{CP}$ characteristic, could be caused by the increase in the IOP as well as the drug-modified cornea surface, biomechanics, and choroidal vasculature.
When discussing our findings, some limitations and other aspects of the presented study should be considered. For instance, a relatively small number of patients were enrolled in the study. Hence, we performed a post-hoc estimation of statistical power. Our analysis, based on the assumption of Gaussianity and a significance level of $5 \%$, was conducted at a statistical power of $90 \%$. For a sample size of fifteen POAG patients included in this study, a decrease of $1.6 \mu \mathrm{m}$ in the mean value of $A_{\mathrm{CP} 1}$ can be differentiated, which translates to a change of $25 \%$. Similarly, we can differentiate an increase of 0.32 in the mean value of $A_{\mathrm{CP} 3 n}$, which is a $50 \%$ change. Detecting $10 \%$ differences in those values would require 94 and 419 patients, respectively. In that sense, our study has a pilot character. Another limitation is the lack of a control group after cataract surgery. However, it can be assumed that the amount of any bias resulting from phacoemulsification with canaloplasty in POAG patients is likely to be the same for all patients and should not affect greatly the main results achieved in the study.

Another limitation of our study is the lack of measuring suture tension during surgery. Such measurement could have added valuable information to the change of the corneal stiffness. Intra-operative non-contact monitoring of canaloplasty procedure and measuring suture tension is challenging, because there is no method to directly measure suture tension during surgery. Recently, intra-operative optical coherence tomography (iOCT) has been reported as a promising noncontact real-time imaging tool during canaloplasty for monitoring the correct implantation and position of the suture into SC. Also, measurements of changes in anterior chamber angle of the steepening of SC enabled assessing suture tensioning indirectly [76]. Once iOCT is not available, suture tension during surgery can be clinically subjectively evaluated by observing the indentation of the trabeculo-Descemet's membrane [77], which has been done, in this study, by the operating surgeon (M. R.). The surgeon has pulled both thread ends synchronously and equally towards each other and perpendicularly to the trabecular meshwork in accordance with "closing a sling" in order to avoid cheese-wiring [78]. It can be assumed that for each patient, during surgery, the suture was knotted under similar tension in order to distend the trabecular meshwork optimally, which in consequence likely results in similar corneal stiffness. Knowledge about the degree of suture tensioning may be helpful in assessing the success of canaloplasty. Also, the amount of SC distention can be assessed post-operatively using anterior imaging techniques, such as high-resolution ultrasound biomicroscopy [4, 40, 79-82] and anterior segment optical coherence tomography [77, 81, 83].

The consequence of the medication washout may also have an impact on the CP parameters, since some topical antiglaucoma medications have been found to modify corneal structure [51, 84] and biomechanics [55-57] as well as 
choroidal circulations $[63,65,66]$. However, the effect of the specific medications taken by patients on the $\mathrm{CP}$ signal characteristics could not be evaluated because of the applied fixedcombination drug therapy and limited sample size.

Summarizing, based on our findings with the animal model, which showed an increase in the power of the third CP harmonic with increasing stiffness of the cornea estimated by Young's modulus [34], we conclude that changes in $A_{\mathrm{CP} 3 \mathrm{n}}$ could reflect alterations in corneoscleral stiffness caused by the surgery. In this sense, $A_{\mathrm{CP} 3 n}$ could be viewed as an indirect supporting indicator for monitoring post-operative corneal biomechanical behavior and for estimating the time at which measures of IOP are no longer biased by the changed cornea boundary conditions caused by canaloplasty.

Acknowledgments The authors would like to thank Prof. D. Robert Iskander for his fruitful comments on an earlier version of the manuscript and Dr. Cezary Sielużycki and Dr. Anna Byszewska for proofreading.

Funding The work of M. E. Danielewska and M. M. Placek has been funded by the National Centre for Research and Development, Poland (grant no. LIDER/074/L-6/14/NCBR/2015). The work of A. K. Kicińska and M. Rękas has been funded by the Science Grant awarded by the Ministry of Science and Higher Education.

\section{Compliance with ethical standards}

Conflict of interest The authors declare that they have no conflict of interest

Ethical approval All procedures performed in studies involving human participants were in accordance with the ethical standards of the Bioethics Committee of the Military Institute of Medicine in Warsaw and with the 1964 Helsinki declaration and its later amendments or comparable ethical standards.

Informed consent Informed consent was obtained from all individual participants included in the study.

Open Access This article is distributed under the terms of the Creative Commons Attribution 4.0 International License (http:// creativecommons.org/licenses/by/4.0/), which permits unrestricted use, distribution, and reproduction in any medium, provided you give appropriate credit to the original author(s) and the source, provide a link to the Creative Commons license, and indicate if changes were made.

\section{References}

1. Khaimi MA (2015) Canaloplasty: a minimally invasive and maximally effective glaucoma treatment. J Ophthalmol 2015:1-5

2. Klink T, Sauer J, Körber NJ et al (2015) Quality of life following glaucoma surgery: canaloplasty versus trabeculectomy. Clin Ophthalmol 9:7

3. Lewis RA, von Wolff K, Tetz M et al (2011) Canaloplasty: threeyear results of circumferential viscodilation and tensioning of Schlemm canal using a microcatheter to treat open-angle glaucoma. J Cataract Refract Surg 37:682-690
4. Brusini P (2014) Canaloplasty in open-angle glaucoma surgery: a four-year follow-up. Sci World J 2014:469609

5. Bull H, von Wolff K, Körber N, Tetz M (2011) Three-year canaloplasty outcomes for the treatment of open-angle glaucoma: European study results. Graefes Arch Clin Exp Ophthalmol 249: $1537-1545$

6. Schoenberg ED, Chaudhry AL, Chod R et al (2015) Comparison of surgical outcomes between phacocanaloplasty and phacotrabeculectomy at 12 months' follow-up. J Glaucoma 24:543549

7. Matlach J, Dhillon C, Hain J et al (2015) Trabeculectomy versus canaloplasty (TVC study) in the treatment of patients with openangle glaucoma: a prospective randomized clinical trial. Acta Ophthalmol 93:753-761

8. Niesel P, Flammer J (1980) Correlations between intraocular pressure, visual field and visual acuity, based on 11 years of observations of treated chronic glaucomas. Int Ophthalmol 3:31-35

9. Ekström C (2009) Elevated intraocular pressure and pseudoexfoliation of the lens capsule as risk factors for chronic open-angle glaucoma. Acta Ophthalmol 71:189-195

10. Konstas AGP, Quaranta L, Mikropoulos DG et al (2012) Peak intraocular pressure and glaucomatous progression in primary openangle glaucoma. J Ocul Pharmacol Ther 28:26-32

11. Silver DM, Farrell RA, Langham ME et al (1989) Estimation of pulsatile ocular blood flow from intraocular pressure. Acta Ophthalmol 67:25-29

12. Liu J, Roberts CJ (2005) Influence of corneal biomechanical properties on intraocular pressure measurement: quantitative analysis. J Cataract Refract Surg 31:146-155

13. Rękas M, Danielewska ME, Byszewska A et al (2016) Assessing efficacy of canaloplasty using continuous 24-hour monitoring of ocular dimensional changes. Invest Ophthalmol Vis Sci 57:2533-2542

14. Zuckerman JL, Taylor KD, Grossman HJ (1977) Noncontact detection of ocular pulse-correlation with carotid stenosis. Invest Ophthalmol Vis Sci 16:1018-1024

15. Northrop RB, Nilakhe SS (1977) A no-touch ocular pulse measurement system for the diagnosis of carotid occlusions. IEEE Trans Biomed Eng 24:139-148

16. Kiel JW (2011) The ocular circulation. In: Colloquium series on integrated systems physiology: from molecule to function. San Rafael, CA: Morgan \& Claypool Life Sciences, 3:1-81

17. Langham ME, Farrell RA, O’Brien Vet al (1989) Blood flow in the human eye. Acta Ophthalmol 67(S191):9-13

18. Ramos RF, Stamer WD (2008) Effects of cyclic intraocular pressure on conventional outflow facility. Invest Ophthalmol Vis Sci 49: 275-281

19. Hashimoto JM, Epstein DL (1980) Influence of intraocular pressure on aqueous outflow facility in enucleated eyes of different mammals. Invest Ophthalmol Vis Sci 19:1483-1489

20. Iskander DR, Kasprzak HT (2006) Dynamics in longitudinal eye movements and corneal shape. Ophthalmic Physiol Opt 26: $572-579$

21. Kowalska MA, Kasprzak HT, Iskander DR et al (2011) Ultrasonic in vivo measurement of ocular surface expansion. IEEE Trans Biomed Eng 58:674-680

22. Danielewska ME, Iskander DR, Krzyżanowska-Berkowska $P$ (2014) Age-related changes in corneal pulsation: ocular dicrotism. Optom Vis Sci 91:54-59

23. Schmetterer LF, Lexer F, Unfried CJ et al (1995) Topical measurement of fundus pulsations. Opt Eng 34:711-717

24. Hommer A, Fuchsjäger-Mayrl G, Resch H et al (2008) Estimation of ocular rigidity based on measurement of pulse amplitude using pneumotonometry and fundus pulse using laser interferometry in glaucoma. Investig Opthalmol Vis Sci 49:4046-4050 
25. Dion C, Singh K, Ozaki T et al (2013) Analysis of pulsatile retinal movements by spectral-domain low-coherence interferometry: influence of age and glaucoma on the pulse wave. PLoS One 8: e54207

26. Singh K, Dion C, Wajszilber M et al (2011) Measurement of ocular fundus pulsation in healthy subjects using a novel Fourier-domain optical coherence tomography. Invest Ophthalmol Vis Sci 52: 8927-8932

27. Kiel JW (1995) The effect of arterial pressure on the ocular pressure-volume relationship in the rabbit. Exp Eye Res 60:267-278

28. Trew DR, James CB, Thomas SHL et al (1991) Factors influencing the ocular pulse - the heart rate. Graefes Arch Clin Exp Ophthalmol 229:553-556

29. Bosley TM, Cohen MS, Gee W et al (1993) Amplitude of the ocular pneumoplethysmography waveform is correlated with cardiac output. Stroke 24:6-9

30. Kasprzak HT, Iskander DR (2007) Spectral characteristics of longitudinal corneal apex velocities and their relation to the cardiopulmonary system. Eye (Lond) 21:1212-1219

31. Danielewska ME, Iskander DR, Kowalska M, Kasprzak HT (2012) Phase dependencies between longitudinal corneal apex displacement and cardiovascular signals: is the ocular pulse influenced by the electrical activity of the heart? Clin Exp Optom 95:631-637

32. Ebneter A, Wagels B, Zinkernagel MS (2009) Non-invasive biometric assessment of ocular rigidity in glaucoma patients and controls. Eye 23:606-611

33. Stalmans I, Harris A, Vanbellinghen V et al (2008) Ocular pulse amplitude in normal tension and primary open angle glaucoma. J Glaucoma 17:403-407

34. Rogala MM, Danielewska ME, Antończyk A et al (2017) In-vivo corneal pulsation in relation to in-vivo intraocular pressure and corneal biomechanics assessed in-vitro. An animal pilot study. Exp Eye Res 162:27-36

35. Licznerski TJ, Jaroński J, Kosz D (2011) Ultrasonic system for accurate distance measurement in the air. Ultrasonics 51:960-965

36. Danielewska ME, Krzyżanowska P, Iskander DR (2014) Glaucomatous and age-related changes in corneal pulsation shape. The ocular dicrotism. PLoS One 9:e102814

37. Evans DW, Hosking SL, Embleton SJ et al (2002) Spectral content of the intraocular pressure pulse wave: Glaucoma patients versus normal subjects. Graefes Arch Clin Exp Ophthalmol 240:475-480

38. Božić M, Dukić ML, Stojković M (2012) Spectral analysis of intraocular pressure pulse wave in open angle glaucomas and healthy eyes. Curr Eye Res 37:1019-1024

39. Battista SA, Lu Z, Hofmann S et al (2008) Reduction of the available area for aqueous humor outflow and increase in meshwork herniations into collector channels following acute IOP elevation in bovine eyes. Investig Opthalmol Vis Sci 49:5346-5352

40. Lewis RA, von Wolff K, Tetz M et al (2007) Canaloplasty: circumferential viscodilation and tensioning of Schlemm's canal using a flexible microcatheter for the treatment of open-angle glaucoma in adults: interim clinical study analysis. J Cataract Refract Surg 33: 1217-1226

41. Bellucci R, Morselli S (2005) Reverse flap dissection for glaucoma surgery. Ophthalmic Surg Lasers Imaging Retina 36:79

42. Kowalska MA, Kasprzak HT, Iskander DR (2009) Comparison of high-speed videokeratoscopy and ultrasound distance sensing for measuring the longitudinal corneal apex movements. Ophthalmic Physiol Opt 29:227-234

43. Kerr J, Nelson P, O'Brien C (1998) A comparison of ocular blood flow in untreated primary open-angle glaucoma and ocular hypertension. Am J Ophthalmol 126:42-51

44. Asejczyk-Widlicka M, Krzyżanowska-Berkowska P, Kowalska M, Iskander DR (2014) Clinical utility of spectral analysis of intraocular pressure pulse wave. BMC Ophthalmol 14:30
45. Pallikaris IG, Kymionis GD, Ginis HS et al (2005) Ocular rigidity in living human eyes. Invest Ophthalmol Vis Sci 46:409-414

46. Dastiridou AI, Ginis HS, De Brouwere D et al (2009) Ocular rigidity, ocular pulse amplitude, and pulsatile ocular blood flow: the effect of intraocular pressure. Invest Ophthalmol Vis Sci 50:5718-5722

47. Karyotakis N (2012) Manometric investigation of the relationship between pulsatile ocular blood flow and intraocular pressure in living human eyes. IOVS 53:ARVO E-Abstract 6862

48. Quaranta L, Manni G, Donato F, Bucci MG (1994) The effect of increased intraocular pressure on pulsatile ocular blood flow in low tension glaucoma. Surv Ophthalmol 38:177-182

49. Tetz M, Koerber N, Shingleton BJ et al (2015) Phacoemulsification and intraocular lens implantation before, during, or after canaloplasty in eyes with open-angle glaucoma. J Glaucoma 24: 187-194

50. Grieshaber MC, Schoetzau A, Flammer J, Orgül S (2013) Postoperative microhyphema as a positive prognostic indicator in canaloplasty. Acta Ophthalmol 91:151-156

51. Konowal A, Morrison J, Brown SV et al (1999) Irreversible corneal decompensation in patients treated with topical dorzolamide. Am J Ophthalmol 127:403-406

52. Wirtitsch MG, Findl O, Kiss B et al (2003) Short-term effect of dorzolamide hydrochloride on central corneal thickness in humans with cornea guttata. Arch Ophthalmol 121:621-625

53. Teus MA, Bolívar G, Alió JL, Lipshitz I (2009) Short-term effect of topical dorzolamide hydrochloride on intrastromal corneal pressure in rabbit corneas in vivo. Cornea 28:206-210

54. Grueb M, Rohrbach JM (2013) Effect of timolol on central corneal thickness. Eur J Ophthalmol 23:784-788

55. Bolívar G, Sánchez-Barahona C, Teus M et al (2015) Effect of topical prostaglandin analogues on corneal hysteresis. Acta Ophthalmol 93:e495-e498

56. Tsikripis P, Papaconstantinou D, Koutsandrea C et al (2013) The effect of prostaglandin analogs on the biomechanical properties and central thickness of the cornea of patients with open-angle glaucoma: a 3-year study on 108 eyes. Drug Des Devel Ther 7:1149-1156

57. Wu N, Chen Y, Yu X et al (2016) Changes in corneal biomechanical properties after long-term topical prostaglandin therapy. PLoS One 11:e0155527

58. Panos GD, Konstantinidis A, Mendrinos E et al (2013) Effect of tafluprost $0.0015 \%$ on central corneal thickness in patients with primary open-angle glaucoma. Curr Eye Res 38:977-982

59. Honda N, Miyai T, Nejima R et al (2010) Effect of latanoprost on the expression of matrix metalloproteinases and tissue inhibitor of metalloproteinase 1 on the ocular surface. Arch Ophthalmol 128: 466-471

60. Wu K-Y, Wang H-Z, Hong S-J (2005) Effect of latanoprost on cultured porcine corneal stromal cells. Curr Eye Res 30:871-879

61. Ito T, Ohguro H, Mamiya K et al (2006) Effects of antiglaucoma drops on MMP and TIMP balance in conjunctival and subconjunctival tissue. Investig Opthalmol Vis Sci 47:823-830

62. Liu Y, Yanai R, Lu Y et al (2006) Effects of antiglaucoma drugs on collagen gel contraction mediated by human corneal fibroblasts. J Glaucoma 15:255-259

63. Schmetterer L, Strenn K, Findl O et al (1997) Effects of antiglaucoma drugs on ocular hemodynamics in healthy volunteers. Clin Pharmacol Ther 61:583-595

64. Rankin SJA, Walman BE, Buckley AR, Drance SM (1995) Color Doppler imaging and spectral analysis of the optic nerve vasculature in glaucoma. Am J Ophthalmol 119:685-693

65. Yoshida A, Feke GT, Ogasawara $\mathrm{H}$ et al (1991) Effect of timolol on human retinal, choroidal and optic nerve head circulation. Ophthalmic Res 23:162-170 
66. Carenini AB, Sibour G, Carenini BB (1994) Differences in the longterm effect of timolol and betaxolol on the pulsatile ocular blood flow. Surv Ophthalmol 38:s118-s124

67. Fuchsjäger-Mayrl G, Wally B, Rainer G et al (2005) Effect of dorzolamide and timolol on ocular blood flow in patients with primary open angle glaucoma and ocular hypertension. Br J Ophthalmol 89:1293-1297

68. Arend O, Harris A, Wolter P, Remky A (2003) Evaluation of retinal haemodynamics and retinal function after application of dorzolamide, timolol and latanoprost in newly diagnosed openangle glaucoma patients. Acta Ophthalmol Scand 81:474-479

69. Affrime MB, Lowenthal DT, Tobert JA et al (1980) Dynamics and kinetics of ophthalmic timolol. Clin Pharmacol Ther 27:471-477

70. Harris A, Shoemaker JA, Burgoyne J et al (1995) Acute effect of topical beta-adrenergic antagonists on normal perimacular hemodynamics. J Glaucoma 4:36-40

71. Harris A, Spaeth GL, Sergott RC et al (1995) Retrobulbar arterial hemodynamic effects of betaxolol and timolol in normal-tension glaucoma. Am J Ophthalmol 120:168-175

72. Harris A, Arend O, Arend S, Martin B (1996) Effects of topical dorzolamide on retinal and retrobulbar hemodynamics. Acta Ophthalmol Scand 74:569-572

73. Harris A, Jonescu-Cuypers CP, Kagemann L et al (2001) Effect of dorzolamide timolol combination versus timolol $0.5 \%$ on ocular bloodflow in patients with primary open-angle glaucoma. Am J Ophthalmol 132:490-495

74. Stewart WC, Garrison PM (1998) $\beta$-blocker-induced complications and the patient with glaucoma. Arch Intern Med 158:221-226

75. Danielewska ME, Messner A, Werkmeister RM, et al (2019) Relationship between the parameters of corneal and fundus pulse signals acquired with a combined ultrasound and laser interferometry technique. Transl Vis Sci Technol 8(4):15. https://doi.org/10. $1167 /$ tvst.8.4.15

76. Siebelmann S, Cursiefen C, Lappas A, Dietlein T (2016) Intraoperative optical coherence tomography enables noncontact imaging during canaloplasty. J Glaucoma 25:236-238
77. Brandao LM, Schötzau A, Grieshaber MC (2015) Suture distension of Schlemm's canal in canaloplasty: an anterior segment imaging study. J Ophthalmol 2015:457605

78. Grieshaber MC, Fraenkl S, Schoetzau A et al (2011) Circumferential viscocanalostomy and suture canal distension (canaloplasty) for whites with open-angle glaucoma. J Glaucoma 20:298-302

79. Lewis RA, von Wolff K, Tetz M et al (2009) Canaloplasty: circumferential viscodilation and tensioning of Schlemm canal using a flexible microcatheter for the treatment of open-angle glaucoma in adults: two-year interim clinical study results. J Cataract Refract Surg 35:814-824

80. Grieshaber MC (2012) Ab externo Schlemm's canal surgery: viscocanalostomy and canaloplasty. In: Developments in ophthalmology. Karger Publishers, Basel, pp 109-124

81. Kuerten D, Plange N, Becker J et al (2018) Evaluation of long-term anatomic changes following canaloplasty with anterior segment spectral-domain optical coherence tomography and ultrasound biomicroscopy. J Glaucoma 27:87-93

82. Doro D, Koerber N, Paolucci P, Cimatti P (2012) Ultrasound biomicroscopy after canaloplasty: clinical study with two different units. Acta Clin Croat 51(Suppl 1):113-118

83. Fuest M, Kuerten D, Koch E et al (2016) Evaluation of early anatomical changes following canaloplasty with anterior segment spectral-domain optical coherence tomography and ultrasound biomicroscopy. Acta Ophthalmol 94:e287-e292

84. Bergonzi C, Giani A, Blini M et al (2010) Evaluation of prostaglandin analogue effects on corneal keratocyte density using scanning laser confocal microscopy. J Glaucoma 19:617-621

Publisher's note Springer Nature remains neutral with regard to jurisdictional claims in published maps and institutional affiliations. 